\title{
A cirurgia ortognática no tratamento da SAHOS: uma revisão de literatura
}

\author{
Orthognathic surgery in the SAHOS' treatment: a literature review \\ Cirugía ortognática en el tratamiento del SAHOS: revisión de la literatura
}

Recebido: 27/12/2020 | Revisado: 27/12/2020 |Aceito: 29/12/2020 | Publicado: 03/01/2021

\author{
Alina Nascimento dos Reis \\ ORCID: https://orcid.org/0000-0002-3239-4871 \\ Universidade Ceuma, Brasil \\ E-mail: alinadosreis@gmail.com \\ Júlio César Silva de Oliveira \\ ORCID: https://orcid.org/0000-0003-2796-5059 \\ Universidade Estadual Paulista "Júlio de Mesquita Filho", Brasil \\ Universidade Ceuma, Brasil \\ E-mail: oliveirajulius@yahoo.com.br \\ Marisa Aparecida Cabrini Gabrielli \\ ORCID: https://orcid.org/0000-0002-7147-1438 \\ Universidade Estadual Paulista "Júlio de Mesquita Filho", Brasil \\ E-mail: marisa.gabrielli@unesp.br \\ Ana Paula Farnezi Bassi \\ ORCID: https://orcid.org/0000-0002-0031-4953 \\ Universidade Estadual Paulista "Júlio de Mesquita Filho", Brasil \\ E-mail: ana.bassi@unesp.br
}

\begin{abstract}
Resumo
A Síndrome da Apneia ou Hipopneia Obstrutiva do Sono (SAHOS) é o distúrbio do sono mais comum na população e é caracterizada por cinco ou mais episódios de obstrução parcial ou total das Vias Aéreas Superiores (VAS) durante o sono, por um período igual ou maior que 10 segundos. Os sintomas mais comuns relatados são sonolência diurna, roncos noturnos, sono agitado e fadiga excessiva, o que interferem diretamente na qualidade de vida do indivíduo. Este trabalho tem como objetivo abordar a cirurgia ortognática como uma opção de tratamento para a Síndrome da Apneia ou Hipopneia Obstrutiva do Sono. Trata-se de uma revisão de literatura onde foi realizada a busca referente ao tema abordado nas bases de dados SCIELO (Scientific Eletronic Library On-line), PubMed, Science Direct e Google Acadêmico, no período de 2013 a 2020. A SAHOS é um grave problema de saúde pública, e cabe também ao cirurgião dentista diagnosticar e tratar, sendo a cirurgia ortognática de avanço maxilomandibular uma alternativa segura e eficaz.
\end{abstract}

Palavras-chave: Apneia obstrutiva do sono; Ronco; Cirurgia ortognática; Avanço mandibular.

\begin{abstract}
Obstructive Sleep Apnea-Hypopnea Syndrome (OSAHS) is the most common sleep disorder in the population and is characterized by five or more episodes of partial or total upper airway (UA) obstruction during sleep, for a period equal to or greater than 10 seconds. The most common reported symptoms are daytime sleepiness, night snoring, restless sleep and excessive fatigue, which directly interfere at the individual's quality of life. This work aims to approach orthognathic surgery as a treatment option for Obstructive Sleep Apnea-Hypopnea Syndrome. It is a literature review where it was performed a search related to the topic covered at the databases SCIELO (Scientific Electronic Library On-line), PubMed, Science Direct and Google Scholar, in the period from 2013 to 2020 . OSAHS is a serious public health problem, and it is also up to the dental surgeon to diagnose and treat, being the advancement maxillomandibular orthognathic surgery a safe and effective alternative.
\end{abstract}

Keywords: Obstructive sleep apnea; Snoring; Orthognathic surgery; Mandibular advancement.

\section{Resumen}

El Síndrome de Apnea-Hipopnea Obstructiva del Sueño (SAHOS) es el trastorno del sueño más común en la población y se caracteriza por cinco o más episodios de obstrucción parcial o total de la vía aérea superior (VAS) durante el sueño, por un período igual o superior a 10 segundos. Los síntomas más comúnmente reportados son somnolencia diurna, ronquidos nocturnos, sueño agitato y fatiga excesiva, que interfieren directamente en la calidad de vida del individuo. El objetivo de este trabajo es abordar la cirugía ortognática como opción de tratamiento para el Síndrome de Apnea-Hipopnea Obstructiva del Sueño. Es una revisión de literatura donde se realiza la búsqueda relacionada con el tema abordado en las bases de datos SCIELO (Scientific Electronic Library On-line), PubMed, Science Direct y Google Scholar, de 2013 a 2020. El SAHOS es un grave problema de salud pública, y también le 
corresponde al cirujano dentista diagnosticar y tratar, y la cirugía ortognática de avance maxilomandibular es una alternativa segura y eficaz.

Palabras clave: Apnea obstructiva del sueño; Ronquidos; Cirugía ortognática; Avance mandibular.

\section{Introdução}

A Síndrome da Apneia ou Hipopneia Obstrutiva do Sono (SAHOS) é um problema de saúde pública de elevada prevalência relacionada a um alto índice de morbimortalidade, e é caracterizada pela interrupção repetitiva total (apneia) ou parcial (hipopneia) das vias aéreas superiores durante o sono, podendo gerar uma má qualidade de vida ao paciente (Panissa, et al., 2018). Os sintomas mais comuns relatados são a sonolência excessiva diurna, fragmentação do sono, roncos, sono agitado, fadiga, além da baixa concentração (Campos et al. 2017; Rubio-Bueno et al. 2018).

A SAHOS é uma condição ainda muito subdiagnosticada e se não for devidamente examinada e tratada pode resultar em danos crônicos à saúde geral do paciente (Panissa et al. 2018). A dificuldade do diagnóstico se deve a privação do sono, muito comum na sociedade atual, o que leva a mascarar alguns dos principais sintomas, como por exemplo, o cansaço diurno. No caso dos roncos, sintoma característico, a dificuldade de diagnóstico se dá quando mesmos só são percebidos se houver alguém para escutá-los. Outra dificuldade é de diferenciar sonolência de depressão, além do caso da obesidade, considerada um dos fatores de risco, já que também está relacionada a sonolência excessiva (Faber et al. 2019; Campos et al. 2017).

A Síndrome da Apneia ou Hipopneia Obstrutiva do Sono possui uma etiologia multifatorial e isso se deve em parte às alterações anatômicas do trato respiratório superior e do esqueleto craniofacial associadas às alterações neuromusculares da faringe e à obesidade (Maahs et al., 2019; Solomon, Veasey \& Rosen, 2019). A literatura descreve como sendo os principais fatores de risco a obesidade, pacientes etilistas, o aumento do tamanho das tonsilas palatinas e faríngeas, a grande circunferência do pescoço, síndromes genéticas com deformidades craniofaciais evidentes e o retrognatismo maxilomandibular, este último sendo uma característica comum encontrada nos pacientes portadores dessa síndrome (Faber et al. 2019; Campos et al. 2017).

O cirurgião-dentista tem a capacidade de diagnosticar a SAHOS através de uma anamnese detalhada com base em todos os fatores de risco e alguns sinais relatados pelo paciente, podendo também detectar deformidades craniofaciais e aspectos anatômicos intraorais que podem contribuir para a diminuição do espaço retropalatal. Além disso, o paciente pode realizar a polissonografia, o exame mais completo para identificação da doença (Poluha, Stefaneli \& Terada 2016).

O tratamento da SAHOS é planejado com base na sua gravidade. Em casos mais leves pode ser indicada a perda de peso ou até o uso de aparelhos intra-orais, contudo, em casos moderados a severos, a cirurgia ortognática de avanço maxilomandibular é uma opção. Essa cirurgia além de promover benefícios estéticos e funcionais ao paciente, tem como objetivo alterar a dimensão das vias aéreas superiores, possibilitando uma melhor passagem de ar e melhor qualidade de vida ao paciente (Maahs et al. 2019).

Dentre os tratamentos que o cirurgião-dentista pode realizar junto ao paciente devidamente diagnosticado com a SAHOS, a cirurgia ortognática ocupa um espaço primordial na qualidade de vida e bem estar, não só do ponto de vista estético, mas sobretudo funcional.

Portanto, objetivou-se uma revisão bibliográfica evidenciando a cirurgia ortognática como opção de tratamento para a Síndrome da Apneia ou Hipopneia Obstrutiva do Sono.

\section{Metodologia}

Este é um estudo de revisão de literatura, descritiva, em que foi realizada uma busca referente ao tema abordado em artigos publicados nas bases de dados SCIELO (Scientific Eletronic Library On-line), PubMed, Science Direct e Google 
Acadêmico, no período de 2013 a 2020. A pesquisa foi realizada utilizando os termos livres e descritores "SAHOS", "Apneia do sono", "Tratamento da apneia", "Orthongatic surgery" e "SAHOS e cirurgia ortognática". Foram incluídos estudos transversais, longitudinais, revisões de literatura e teses em português e inglês.

Selecionou-se inicialmente todos os artigos originais dando prioridade aos publicados no período de 2013 a 2020 relacionados à Cirurgia Ortognática no tratamento da Apneia ou Hipopneia Obstrutiva do Sono, e foram descartados aqueles que não condiziam com o tema proposto.

\section{Resultados e Discussão}

O sono é um fenômeno essencial para o bem estar do indivíduo e fundamental para uma boa saúde mental e emocional, e é por meio dele que todo o organismo e o Sistema Nervoso Central (SNC) são renovados (Dal Fabbro, Júnior \& Tufik, 2010). Ito em 2005, definiu o sono como sendo um "processo dinâmico e fisiológico de perda de consciência e inativação da musculatura voluntária" sendo reversível frente à estímulos. No entanto, certos estímulos não são suficientes para nos mantermos acordados e geram somente microdespertares, os quais acabam levando à uma má qualidade do sono (Pacheco, Anjos \& Maya, 2015).

A Síndrome da Apneia ou Hipopneia Obstrutiva do Sono (SAHOS) constitui um dos distúrbios respiratórios que se manifesta em alguns indivíduos ao dormir e que interfere diretamente na sua qualidade de vida (Pacheco et al. 2015). É uma condição que afeta mais homens do que mulheres, não se sabe ao certo o motivo, mas diferenças sociais, anatômicas e hormonais são levadas em consideração. Alguns estudos apontam que após a menopausa as mulheres passam a ter o mesmo risco dos homens para desenvolver a doença (Tanna et al. 2016; Maahs et al. 2019), sugerindo que a progesterona seja responsável por influenciar na atividade dilatadora dos músculos das vias aéreas superiores (VAS), protegendo as mulheres da SAHOS, enquanto os hormônios masculinos favorecem o aparecimento da doença (Galtieri et al. 2019; Pacheco et al. 2015). Pode-se estimar que há uma prevalência na população geral em $9 \%$ a 35\%, porém, existe uma taxa de subdiagnóstico que pode alcançar 90\% do total (Almeida et al. 2019).

Essa dificuldade de diagnóstico se deve ao fato da possibilidade de o paciente negar ou minimizar os roncos, sendo estes somente percebidos se tiver alguém para escutá-los. Além disso, considerando que a privação do sono está cada vez mais comum na sociedade, há uma dificuldade de diferenciar a sonolência diurna característica da SAHOS, com fadiga e/ou depressão, e até mesmo relacionar os sintomas de cansaço com a obesidade (Campos et al. 2017).

Sua etiologia é multifatorial e pode variar devido às alterações exógenas, patológicas e alterações anatômicas no esqueleto craniofacial associadas à alteração neuromuscular da faringe, além de obesidade, alterações endócrinas, hipotonicidade da musculatura (devido ao consumo de álcool e relaxantes musculares), posição de decúbito dorsal e fatores genéticos (Galtieri et al. 2019; Poluha et al. 2016).

A maioria das causas está associada a alterações anatômicas das vias aéreas superiores, como por exemplo o retrognatismo mandibular e a hipoplasia maxilar (alterações no desenvolvimento da maxila) o que resulta na diminuição do espaço retropalatino. Há ainda o desvio de septo, hiperplasia de amigadas e adenoides, alongamento do palato mole, estreitamento da válvula nasal externa e posição do osso hioide mais anterior e inferior. Quanto maior a distância do osso hioide ao plano mandibular, maior a severidade da SAHOS. Além disso, pacientes com face longa possuem as vias aéreas mais estreitas, dificultando a passagem de ar (Trindade, 2019; Maahs et al., 2019; Gottsauner-Wolf, Laimer \& Bruckmoser, 2018).

O maior fator de risco para pacientes com a SAHOS, tanto no sexo masculino como no sexo feminino, e que abrange $70 \%$ dos casos, é a obesidade. Isso se deve aos tecidos faríngeos terem maior deposição de gordura causando excesso de tecido e um maior risco de obstrução e colapso das vias aéreas superiores (Maahs et al. 2019). Ressalta-se que, com a obesidade há 
um aumento da circunferência do pescoço (CP), de valores superiores a $40 \mathrm{~cm}$ para homens e $35 \mathrm{~cm}$ para mulheres, o que apresenta uma importante correlação com o distúrbio respiratório por ser um marcador de deposição de gordura e importante preditor de roncos (Schmidt, et al. 2019; De Sá Catão et al. 2015).

Outros fatores de risco também podem ser identificados, como o tabagismo que além de influenciar diretamente no sono por proporcionar uma maior dificuldade de adormecer e de manter o sono contínuo durante a noite, pode ainda ser considerado um fator predisponente de doenças pulmonares e cardiovasculares. O efeito sinérgico da SAHOS com a doença cardiovascular pode resultar no aumento da pressão arterial, desenvolvimento de aterosclerose, além de aumentar o risco da morbimortalidade. Estudos mostram que indivíduos tabagistas apresentam três vezes mais chances de apresentarem a SAHOS (Alves, Silva \& Furlan 2020).

Sendo um dos mais comuns distúrbios do sono, a SAHOS é caraterizada pela recorrência de cinco ou mais episódios de obstrução total (apneia) ou parcial (hipopneia) por no mínimo 10 segundos do fluxo de ar das vias aéreas superiores (Trindade, 2019; Tanna et al. 2020). A falta do fluxo de ar se dá pela faringe que oclui, e então os movimentos do tórax e do abdômen ficam diminuídos e/ou opostos, havendo uma diminuição dos níveis de oxigênio no sangue (hipoxemia) e um aumento dos níveis de gás carbônico (hipercapnia). Para que o sangue possa chegar aos pulmões e as trocas gasosas serem realizadas, ocorre um aumento do trabalho cardíaco com o intuito de reestabelecer os níveis de oxigênio. Esse processo faz com que o indivíduo precise de mais esforço respiratório, levando à alteração e irregularidade do sono, dificuldade de adormecer e roncos (Maahs et al. 2019; Campos et al. 2017; Pacheco 2015).

Segundo a Associação Brasileira do Sono, as manifestações clínicas mais comuns da SAHOS são: ronco, tosse, alterações cognitivas, queda do rendimento intelectual, sonolência diurna, cefaleia matinal, apneia presenciada, engasgos, boca seca, irritabilidade, dispneia e impotência (Barreto 2017).

A SAHOS, quando não tratada, pode trazer graves consequências para o indivíduo, como alterações cardiovasculares e metabólicas, sendo preocupante devido ao alto grau de letalidade, hipertensão arterial sistêmica (HAS), aumento de risco de acidentes automobilísticos, arritmias cardíacas, doenças coronariana isquêmica, acidente vascular encefálico (AVE), insuficiência cardíaca, além da redução da qualidade do sono e da qualidade de vida (Faber et al. 2019; Schmidt et al. 2019).

Para um correto diagnóstico da síndrome é necessária uma anamnese detalhada e um exame físico e clínico minuciosos que devem contemplar as variáveis antropométricas (peso e altura), o índice de massa corporal (IMC), a medida da circunferência do pescoço (na borda anterior da membrana cricotireoidea), mensuração da pressão arterial e avaliação das vias aéreas superiores. Além disso, é imprescindível avaliar o paciente como um todo e identificar suas queixas com relação ao cansaço excessivo diurno, roncos, sensação de boca seca, irritabilidade, falta de concentração, despertares noturnos ou sono agitado (Barreto 2017; Strassburger \& Bauer 2019; Schmidt 2019; Tanna et al. 2016).

O cirurgião-dentista possui um importante papel no diagnóstico uma vez que está em contato direto com a cavidade bucal e o esqueleto facial. É necessário realizar uma avaliação completa de toda a morfologia crâniofacial para a identificação de alterações tanto da mandíbula como da maxila, bem como alterações de oclusão, como por exemplo, pacientes classe II, pacientes com mordida aberta, mordida cruzada, atresia maxilar e palato ogival (Dalla Torre et al. 2017).

Na realização do exame intraoral, também pode ser utilizada a classificação de Mallampati modificada, onde se pode determinar a proporção dos tecidos moles da cavidade bucal com a orofaringe. Essa avaliação é realizada com o paciente sentado com a boca em abertura máxima e língua relaxada buscando observar o espaço da orofaringe (Maahs et al. 2019; Tanna et al. 2016). A classificação varia de I a IV, sendo Classe I, palato mole, fauce, úvula e pilares amigdalianos são visíveis; na Classe II, palato mole, fauce e úvula são visíveis; na Classe III, o palato mole e a base da úvula são visíveis; e na Classe IV, o palato mole não é totalmente visível. Sendo as classes III e IV consideradas desfavoráveis (Cunha et al. 2020). 
Ainda relacionado ao exame intraoral dos tecidos moles, além de observar o espaço da orofaringe, é importante o cirurgião-dentista observar no momento da anamnese o tamanho da língua e sua posição, bem como o tamanho das amigdalas e adenoides, tendo em vista que quando estão com o seu tamanho aumentado, dificultam a passagem de ar. Também é importante descobrir a presença de certas condições, como hipotireoidismo, acromegalia, obesidade, Síndrome de Down e alterações genéticas que podem promover o estreitamento da VAS (Schmidt 2019; Faber et al. 2019).

O exame mais completo para o diagnóstico da SAHOS, e um dos mais utilizados, é a polissonografia de noite inteira que é realizada em centros especializados de estudo do sono, onde é registrado o fluxo de ar, os níveis de oxigênio no sangue, o esforço respiratório, a atividade elétrica do coração (eletrocardiograma), dos olhos (eletrooculograma), do cérebro (eletroencefalograma) e do músculo esquelético (eletromiograma). Com isso, o exame consegue identificar e quantificar os episódios de apneia e hipopneia que acontecem por hora (Biedermann 2020; Poluha et al. 2016).

Os resultados do exame irão determinar o índice de Apneia/Hipopneia (IAH) e a gravidade da doença. A SAHOS pode ser considerada leve quando o IAH varia entre 5 a 15 pausas respiratórias por hora durante o sono, ela é moderada quando o IAH é entre 15 e 30 pausas respiratórias por hora, e grave quando o IAH é maior que 30 episódios (Schwartz et al. 2018; Tanna et al. 2016).

Devido ao alto custo da polissonografia e por esse exame gerar, em alguns casos, desconforto aos pacientes, foram criados questionários para facilitar no diagnóstico e avaliar o sono destes indivíduos. A Escala de Sonolência de Epworth (Epworth Sleepiness Scale - ESS) é um dos questionários de fácil aplicação, rápido e sem qualquer custo, que consiste em 8 questões com um score de 0 a 3 sobre a possibilidade de o paciente adormecer em determinadas situações (Bom-fim et al. 2019). Outra opção é o Questionário de Berlin (QB) que se divide em três categorias, onde a categoria 1 é com relação à presença de ronco, a categoria 2 sobre episódios de sonolência diurna, e a categoria 3 sobre histórico de Hipertensão Arterial Sistêmica (HAS) e cálculo de IMC (Barreto 2017; Campos et al. 2017; Araújo-Melo et al. 2016).

Outro exame que pode ser realizado é a Cefalometria sobre telerradiografias laterais, que é o método mais utilizado para medir características craniofaciais e sua relação com a SAHOS (Galtieri et al. 2019). Além de auxiliar no plano de tratamento, ela oferece uma visualização bidimensional das estruturas anatômicas e possibilita a identificação de alterações craniofaciais. Pode-se avaliar ainda o espaço posterior da via aérea superior, posição do osso hioide, tamanho do palato mole, maxila e mandíbula, além de ser essencial no acompanhamento pós-cirúrgico da cirurgia ortognática. Contudo, ela se mostra limitada por reproduzir apenas em planos bidimensionais (Macedo 2011; Poluha et al. 2016; Oslen 2019).

As opções de tratamento da SAHOS variam de acordo com sua gravidade. As mais conservadoras são a perda de peso, a higiene do sono que busca incluir medidas para modificar hábitos inadequados com relação ao sono, o uso de injetores de ar comprimidos, que são os aparelhos de CPAP (Continuous positivee airway pressure) e BIPAP (bilevel positive pressure airway) com pressão aérea usados com uma máscara nasal durante o sono, que por sua vez são aparelhos de controle e não de cura. Há ainda o uso de aparelhos intrabucais que atuam prevenindo o colapso entre os tecidos da orofaringe e da base da língua (Poluha et al. 2016; Barreto 2017).

Em casos onde o paciente não se adaptou às opções mais conservadoras ou onde a origem do problema é devido a alguma anormalidade, podem ser indicados tratamentos cirúrgicos como adenoamigdalectomia, septoplastia, turbinectomia, uvulopalatofaringoplastia, glossectomia e a cirurgia ortognática (Poluha et al. 2016; Prado et al. 2017).

A cirurgia ortognática no tratamento da SAHOS é um dos procedimentos mais eficazes para a expansão da via aérea superior. É indicado para adultos e adolescentes com a ossificação completa e portadores de SAHOS moderada a severa, deficiência mandibular, obesidade, pacientes onde a saturação de oxigênio é inferior a 70\%, e em casos de insucesso dos tratamentos anteriores. Além disso, para a eficácia do tratamento e escolha da cirurgia, deve sempre levar em consideração a 
oclusão e a estética do paciente (Rossi et al. 2019; Solomon et al. 2019; Barreto 2017; Camacho, Jacobson \& Schendel 2013). Estudos mostram que a maioria dos pacientes submetidos a esse tipo de procedimento possuem resultado a curto prazo de redução satisfatória do IAH, se mantendo estável a longo prazo (Jaspers et al. 2013).

A movimentação cirúrgica dos maxilares realizada a partir da cirurgia ortognática tem por objetivo modificar estruturas anatômicas e por consequência aumentar o espaço aéreo faríngeo. A via aérea sofre modificações devido aos ossos, músculos e ligamentos estarem conectados entre si e as várias estruturas como a base da língua, osso hioide, paredes faríngeas e palato mole. Com isso, o avanço maxilomandibular (MMA) acaba carregando a musculatura da língua e da região supra hioidea, bem como acarreta um reposicionamento do véu palatino e dos músculos da região gerando um aumento do espaço retropalatino (Oslen 2019; Pinto et al. 2013).

A técnica da cirurgia ortognática para esses casos consiste em um avanço maxilomandibular (MMA) através de uma osteotomia do tipo Le Fort I e uma osteotomia sagital retromolar bilateral na mandíbula (Vigneron et al. 2017). Mais recentemente, além do avanço da maxila e mandíbula, foi-se adicionada à técnica uma rotação anti-horária do plano oclusal mandibular (Zinser, Zachow \& Sailer. 2013; Frey, Gabrielova \& Gladilin 2018).

Na primeira etapa do procedimento, com o paciente sob anestesia geral e intubação nasotraquel, é realizada uma osteotomia Le Fort I iniciando pela abertura piriforme seguindo até o pilar zigomático. O corte posterior é realizado através da tuberosidade terminando abaixo do processo pterigoide do osso esfenoide e uma osteotomia em forma de $\mathrm{V}$ no septo nasal para evitar a sua compressão. Em seguida, a osteotomia sagital mandibular é realizada e fixada com mini placas e parafusos de titânio. O avanço maxilomandibular deve ser de aproximadamente 10 a $12 \mathrm{~mm}$, o que vai permitir o aumento do espaço retropalatal, diminuindo ou impedindo o colapso das vias aéreas superiores (Frey et al. 2018; Rossi et al. 2019).

Adicionada a esta técnica há ainda a rotação bimaxilar, onde após as osteotomias da maxila e mandíbula, realiza-se uma leve rotação da maxila no sentindo anti-horário com a mandíbula sendo posicionada acompanhando o plano oclusal maxilar e um pouco mais para a anterior. Isso resulta em um aumento significativo das vias aéreas superiores alterando a forma geométrica redonda da faringe para uma forma mais elíptica e levando um posicionamento mais anterior e superior do osso hioide. Há ainda uma melhora na fisionomia do paciente que se apresenta mais harmônica, onde a posição anterior da maxila permanece praticamente a mesma quando a técnica de rotação é utilizada, melhorando assim a estética com a diminuição do ângulo nasolabial e do lábio superior, ao contrário do que ocorre quando realizada técnica convencional de AMM, onde a maxila deve ser posicionada anteriormente em até $10 \mathrm{~mm}$ (Vigneron et al. 2017; Zinser et al. 2013; Araújo 2018).

\section{Considerações Finais}

Tendo em vista que a Síndrome da Apneia ou Hipopneia Obstrutiva do Sono é um grave problema de saúde pública com elevada morbimortalidade e que atinge uma grande parte da população, a cirurgia ortognática de avanço maxilomandibular adicionada a técnica de rotação anti-horária é uma opção de tratamento que se mostra eficaz a longo prazo. Com isso, há uma ampliação da via aérea superior e da orofaringe possibilitando uma melhor passagem de ar, além de uma melhora na estética facial e na qualidade de vida do paciente.

\section{Referências}

Almeida, A., Santos, A., Teixeira, F., Drummond, F., Órfão, R., Moreira, S., Sousa, S., \& Taleço, T. (2019). Documento de Consenso na Abordagem PeriOperatória do Doente com Síndrome da Apneia Obstrutiva do Sono. Revista da Sociedade Portuguesa de Anestesiologia.28(4) $238-248$.

Alves, J. C., Silva, J. K. L. S. da, \& Furlan, S. F. (2020). Apneia obstrutiva do sono e tabagismo. Revista de Medicina, 99(2), 164-169.

Araújo, R. S. M. (2018) Diagnóstico e Tratamento da Síndrome da Aapneia-Hipopneia Obstrutiva do Sono no âmbito da Medicina Dentária. Dissetação de Mestrado. Instituto Universitário Egas Moniz. 
Araújo-Melo, M. H., Neves, D. D., Ferreira, L. V. M. V., Moreira, M. L. V., Nigri, R., \& Simões, S. M. G. (2016). Questionários e Escalas úteis na pesquisa da Síndrome da Apneia Obstrutiva do Sono. Revista Hospital Universitário Pedro Ernesto, 15(1), 48-54.

Barreto, D. A. (2017). A Síndrome da Apneia/Hipopneia Obstrutiva do Sono na Perspetiva do Médico Dentista. Dissertação de Mestrado, Universidade Fernando Pessoa, Porto.

Biedermann (2020). Síndrome da Apneia-Hipopneia Obstrutiva do Sono: Tratamento com aparelhos intra-orais de avanço mandibular. Dissertação de mestrado. Universidade Fernando Pessoa, Porto.

Bom-fim, H. J. J. (2019). Validação da Escala de Sonolência Epwort na Queixa do Sono em diferentes amostras da população Portuguesa segundo o modelo de Rasch. Dissertação de Mestrado, Universidade Lusófona, Lisboa.

Camacho, M., Jacobson, R. L., \& Schendel, S. A. (2013). Surgical treatment of obstructive sleep apnea. Sleep Medicine Clinics, 8(4), $495-503$.

Campos, C. O. C., Soares, Y. P., Colaço, P. X. A., \& Cruz, B. M. S. (2017) Síndrome da Apneia Obstrutiva do Sono. Revista Inspirar Movimento \& Saúde, $12(41), 29-36$.

Cunha, T. C. A., Guimarães, T. M., Almeida, F. R., Haddad, F. L. M., Godoy, L. B. M., Cunha, T. M., Silva, L. O., Tufik, S., \& Bittencourt, L. (2020). Using craniofacial characteristics to predict optimum airway pressure in obstructive sleep apnea treatment. Brazilian Journal of Otorhinolaryngology, 86(2), 174179.

Dal Fabbro, C., Júnior, C. M. C., \& Tufik, S. (2010). A odontologia na medicina do sono. Maringá: Dental Press.

Dalla Torre, D., Burtscher, D., Widmann, G., Rasse, M., Puelacher, T., \& Puelacher, W. (2017). Long-term influence of mandibular advancement on the volume of the posterior airway in skeletal Class II-patients: a retrospective analysis. British Journal of Oral and Maxillofacial Surgery, 55(8), 780-786.

De Sá Catão, C. D., Freitas, V. J. G., Alves, L. S. B., Cruz, J. B. da, Ribeiro, A. I. A. M., \& Macena, M. C. B. (2015). Fatores de risco para a síndrome da apneia e hipopneia obstrutiva do sono em docentes de odontologia. Revista Da Faculdade de Odontologia - UPF, 20(2), 145-149.

Faber, J., Faber, C, \& Faber, A. P. (2019) Obstructive Sleep Apnea in Adults. Dental Press J Orthod. 24(3):99-109.

Frey, R., Gabrielova, B., \& Gladilin, E. (2018). A combined planning approach for improved functional and esthetic outcome of bimaxillary rotation advancement for treatment of obstructive sleep apnea using 3D biomechanical modeling. PLoS ONE, 13(8), 1-15.

Galtieri, R. M. S., Salles, C., Melo, A., \& Souza-Machado, A. (2019). Tipos craniofaciais e relação com a síndrome da apneia obstrutiva do sono. Revista de Ciências Médicas e Biológicas, 18(3), 380.

Gottsauner-Wolf, S., Laimer, J., \& Bruckmoser, E. (2018). Posterior Airway Changes Following Orthognathic Surgery in Obstructive Sleep Apnea. Journal of Oral and Maxillofacial Surgery, 76(5), 1093.e1-1093.e21.

Jaspers, G. W., Booij, A., De Graaf, J., \& De Lange, J. (2013). Long-term results of maxillomandibular advancement surgery in patients with obstructive sleep apnoea syndrome. British Journal of Oral and Maxillofacial Surgery, 51(3), e37-e39.

Maahs, M. A. P., Maahs, T. P., \& Maahs, G. S. (2019). Fatores de risco à síndrome da apneia obstrutiva do sono no adulto. Revista de Ciências Médicas e Biológicas, 18(2), 266.

Macedo, I. A. B. (2011). Avaliação da qualidade de vida e de fatores odontológicos relacionados com a síndrome da apneia obstrutiva do sono. Dissertação de Mestrado. Universidade Tiradentes, Aracajú.

Oslen, B. R. N (2019) Influência da cirurgia ortognática na via aérea superior: uma avaliação tridimensional retrospectiva. Dissertação de Mestrado, Universidade de Cuiabá.

Pacheco, F., Dos Anjos, S. E., \& Maia, F. B. E. (2015). Síndrome Da Apnéia / Hipopnéia Obstrutiva Do Sono : Artigo De Revisão Bibliográfica. Rev UNILUS Ensino e Pesquisa, 12(29), 45-52.

Panissa, C., Morawski, R., Tonietto, L., Silveira, V. S., Gulinelli, J. L., \& Calcagnotto, T. (2018). Cirurgia ortognática para tratamento da síndrome de apneia obstrutiva do sono: relato de caso. Revista Da Faculdade de Odontologia - UPF, 22(3), 337-341.

Pinto, L., Vargas, B., Ramos, V., Coutinho, M., \& Faria, K. (2013). Avanço maxilomandibular no tratamento da Síndrome da Apneia e Hipopneia Obstrutiva do Sono. Revista de Cirurgia e Traumatologia Buco-Maxilo-Facial, 13(1), 09-12.

Poluha, R. L., Stefaneli, E. Á. B., \& Terada, H. H. (2016). A Odontologia na sindrome da apneia obstrutiva do sono: diagnóstico e tratamento. Revista Brasileira de Odontologia, 72(1/2), 87.

Prado, B. N., Fernandes, E. G., Moreira, T. C. A., \& Gavranich Jr, J. (2017). Apneia Obstrutiva do Sono: diagnóstico e tratamento. Revista de Odontologia Da Universidade Cidade de São Paulo, 22(3), 233.

Rossi, D. S., Romano, M., Sweed, A. H., Baj, A., Gianni, A. B., \& Beltramini, G. A. (2019). Use of CAD-CAM technology to improve orthognathic surgery outcomes in patients with severe obstructive sleep apnoea syndrome. Journal of Cranio-Maxillofacial Surgery, 47(9), 1331-1337.

Rubio-Bueno, P., Moreno, A. C., Landete, P., Zamora, E., Wix, R., Ancochea, J., \& Naval-Gias, L. (2018). Obstructive sleep apnoea: An innovative minimally invasive approach using mandibular branch distraction. Revista Espanola de Cirugia Oral y Maxilofacial, 40(2), 55-64.

Schmidt, L., Diane, B. M., Ademar, C. A., Soder, F. T., \& Benetti, F. (2019). Apneia obstrutiva do sono e doenças crônicas não transmissíveis. Revista da Associação Brasileira de Nutrição. Estado de Säo Paulo, 7894, 155-159. 
Research, Society and Development, v. 10, n. 1, e6110111524, 2021

(CC BY 4.0) | ISSN 2525-3409 | DOI: http://dx.doi.org/10.33448/rsd-v10i1.11524

Schwartz, M., Acosta, L., Hung, Y. L., Padilla, M., \& Enciso, R. (2018). Effects of CPAP and mandibular advancement device treatment in obstructive sleep apnea patients: a systematic review and meta-analysis. Sleep and Breathing, 22(3), 555-568.

Solomon, C. G., Veasey, S. C., \& Rosen, I. M. (2019). Obstructive sleep apnea in adults. New England Journal of Medicine, 380(15), $1442-1449$.

Strassburguer, C. S., \& Bauer, L. G. (2019) Conhecimentos e Práticas dos Cirurgiões Dentistas de Porto Alegre sobre a Apneia Obstrutiva do Sono. Trabalho de conclusão de curso, Universidade Federal do Rio Grande do Sul.

Tanna, N., Smith, B. D., Zapanta, P. E., Karanetz, I., Andrews, B. T., Urata, M. M., \& Bradley, J. P. (2016). Surgical management of obstructive sleep apnea. Plastic and Reconstructive Surgery, 137(4), 1263-1272.

Trindade, P. A. K. (2019). Eficácia do Avanço Maxilo-Mandibular do tratamento da Síndrome da Apneia Obstrutiva do Sono: Revisão de Literatura. Dissertação de Mestrado. Universidade Estadual Paulista, São Paulo.

Vigneron, A., Tamisier, R., Orset, E., Pepin, J. L., \& Bettega, G. (2017). Maxillomandibular advancement for obstructive sleep apnea syndrome treatment: Long-term results. Journal of Cranio-Maxillofacial Surgery, 45(2), 183-191.

Zinser, M. J., Zachow, S., \& Sailer, H. F. (2013). Bimaxillary "rotation advancement" procedures in patients with obstructive sleep apnea: A 3-dimensional airway analysis of morphological changes. International Journal of Oral and Maxillofacial Surgery, 42(5), 569-578. 